Mens

Revue d'histoire intellectuelle et culturelle

mens

\title{
En filigrane : l'Empire britannique comme toile de fond de la commission Laurendeau-Dunton et des années 1960
}

\section{Srilata Ravi et Claude Couture}

Volume 14-15, numéro 2-1, printemps-automne 2014

La Commission royale d'enquête sur le bilinguisme et le biculturalisme a 50 ans : période révolue ou projet inachevé ?

URI : https://id.erudit.org/iderudit/1035530ar

DOI : https://doi.org/10.7202/1035530ar

Aller au sommaire du numéro

Éditeur(s)

Centre de recherche en civilisation canadienne-française

ISSN

1492-8647 (imprimé)

1927-9299 (numérique)

Découvrir la revue

Citer cet article

Ravi, S. \& Couture, C. (2014). En filigrane : l'Empire britannique comme toile de fond de la commission Laurendeau-Dunton et des années 1960. Mens,

14-15(2-1), 175-201. https://doi.org/10.7202/1035530ar

\section{Résumé de l'article}

Dans cet article, nous soutenons que la Commission royale d'enquête sur le bilinguisme et le biculturalisme a été le produit d'un contexte marqué par la fin de l'Empire britannique et une réponse aux demandes des Canadiens français concernant l'attachement à cet Empire manifesté par certains Canadiens britanniques après la Seconde Guerre mondiale. Nous pensons que, du Canada colonial britannique au Canada postcolonial d'aujourd'hui, il y a une étonnante continuité culturelle et politique. Nous avançons que la fin de l'Empire a eu des effets significatifs au Canada britannique dans les années 1950 et 1960, et que cette toile de fond n'a pas entièrement disparu. Afin justement de contribuer à mieux comprendre le contexte des années 1960, nous proposons un bref aperçu de la littérature récente sur l'Empire britannique, de la place que les Canadiens français occupent dans cet Empire à la veille de la Commission royale d'enquête sur le bilinguisme et le biculturalisme et un bilan de ce qui nous apparaît comme la résistance toujours actuelle à l'expansion des droits des francophones dans un contexte pourtant postcolonial, et ce, depuis un demi-siècle. 


\title{
En filigrane : l'Empire britannique comme toile de fond de la commission Laurendeau-Dunton et des années 1960
}

\author{
Srilata Ravi et Claude Couture \\ Université de l'Alberta
}

\section{Résumé}

Dans cet article, nous soutenons que la Commission royale d'enquête sur le bilinguisme et le biculturalisme a été le produit d'un contexte marqué par la fin de l'Empire britannique et une réponse aux demandes des Canadiens français concernant l'attachement à cet Empire manifesté par certains Canadiens britanniques après la Seconde Guerre mondiale. Nous pensons que, du Canada colonial britannique au Canada postcolonial d'aujourd'hui, il y a une étonnante continuité culturelle et politique. Nous avançons que la fin de l'Empire a eu des effets significatifs au Canada britannique dans les années 1950 et 1960, et que cette toile de fond n'a pas entièrement disparu. Afin justement de contribuer à mieux comprendre le contexte des années 1960, nous proposons un bref aperçu de la littérature récente sur l'Empire britannique, de la place que les Canadiens français occupent dans cet Empire à la veille de la Commission royale d'enquête sur le bilinguisme et le biculturalisme et un bilan de ce qui nous apparaît comme la résistance toujours actuelle à l'expansion des droits des francophones dans un contexte pourtant postcolonial, et ce, depuis un demi-siècle. 


\section{Abstract}

In this article, we propose that the Commission on Bilingualism and Biculturalism is best understood in the context of the End of Empire and that it was in particular a response to the demands of French Canadians in the face of postwar British Canadian attachment to the Empire. We believe that a remarkable cultural and political continuity exists between colonial British Canada and today's postcolonial Canada. We argue that the End of Empire backdrop had a significant impact in British Canada in the 1950s and 1960s and that this backdrop has not entirely disappeared. To better understand the context of the 1960s, we propose a brief survey of recent scholarship on the British Empire and of French Canada's place in the Empire on the eve of the Commission's work, and we contemplate the continued resistance, fifty years on, to the expansion of Francophone rights in circumstances that are nonetheless postcolonial.

Dans le journal qu'il a tenu pendant les travaux de la Commission royale d'enquête sur le bilinguisme et le biculturalisme, André Laurendeau écrivait : "Ceci nous entraîne à parler de Trois-Rivières, où $4 \%$ de la population réussit à obliger l'immense majorité des Trifluviens à savoir l'anglais s'ils veulent occuper des postes supérieurs. Je souligne qu'il s'agit là d'une situation nettement coloniale $[\ldots]^{1}$. " Par ailleurs, selon François Charbonneau : "L'après-guerre enclenche aussi un mouvement mondial de décolonisation qui marque la fin de l'Empire britannique. Pendant un bref moment, les "Canadiens anglais " étaient d'une certaine manière des impérialistes sans empire $^{2}$. " Nous proposons d'explorer cette dimension coloniale, selon nous oubliée, en passant en revue plusieurs ouvrages récents portant sur le Canada et les années 1960, de même que l'importante

1 André Laurendeau, Journal tenu pendant la Commission royale d'enquête sur le bilinguisme et le biculturalisme, Montréal, VLB éditeur, 1990, p. 177.

2 François Charbonneau, "Comprendre le nouveau nationalisme canadien : le Canada comme idéal moral et politique ", Argument, vol. 7, n 1 (automne 2004-hiver 2005), p. 40. 
littérature des dernières décennies traitant de l'Empire britannique. Dans cet article, les années 1960 sont considérées comme une période charnière et les travaux de la commission LaurendeauDunton comme un produit issu de cette période de basculement d'un monde colonial à un monde postcolonial, sans que certains aspects de la période coloniale disparaissent complètement. Nous terminons l'article par une série d'exemples montrant, selon nous, les traces encore présentes de la période coloniale britannique dont les effets négatifs, notamment vis-à-vis des francophones, sont toujours présents dans la culture politique hégémonique au Canada, malgré le renouvellement apparent des identités canadiennes dans les années 1960.

\section{L'historiographie canadienne et les années 1960}

Aujourd'hui, notamment dans la littérature en langue anglaise produite au Canada, la dimension coloniale du passé canadien est souvent évoquée de telle sorte que l'Empire britannique, pourtant omniprésent, reste une abstraction. Les deux colonialismes, britannique et français, sont aussi souvent décrits comme étant égaux, et le français apparaît de plus en plus dans la littérature contemporaine comme strictement "l'autre " langue coloniale, servant même d'instrument de discrimination raciale entre les colonisateurs français et britanniques, d'une part, et les Autres, d'autre part. Toute notion de hiérarchie entre les colonisateurs, attribuable notamment à la Conquête, est écartée, et le fait que l'élément francophone ait pu résister aux tendances à l'uniformisation de la culture et de la langue anglaise est rarement reconnu. Ainsi, dans deux ouvrages récents, Sean Mills et Bryan Palmer évoquent, de façon contradictoire, cette dimension de l'héritage colonial au Canada. Dans The Empire Within', l'auteur, Sean Mills, parle d'un empire intérieur sans jamais, selon nous, le définir clairement, sans

${ }^{3}$ Sean Mills, The Empire Within: Postcolonial Thought and Postcolonial Activism in Sixties Montreal, Montréal, McGill-Queen's University Press, 2010. 
référence, non plus, très claire à l'Empire britannique et sans évoquer la situation des minorités francophones hors Québec, pourtant une des préoccupations importantes de l'époque. Aucun lien n'est établi avec la Révolution tranquille qui se déroule au même moment en Acadie sous le gouvernement de Louis Robichaud ni, essentiellement, avec les luttes menées au Québec en dehors de Montréal, à cette époque. Dans ce livre, n'existent, en quelque sorte, ni le Québec hors de Montréal ni la francophonie hors du Québec.

Dans Canada's 1960s: The Ironies of Identity in a Rebellious Era ${ }^{4}$, l'historien Bryan Palmer prend un point de vue différent en s'inspirant de la théorie marxiste pour répondre à certaines analyses postmodernes et isoler ce qu'il décrit comme étant des situations ironiques et déstabilisantes, notamment les turbulentes années 1960. Selon lui, le Canada aurait connu plusieurs de ces situations ironiques au cours de cette décennie et aurait basculé d'une identité britannique à une situation marquée par l'incertitude sans cesse renouvelée. Le mouvement ouvrier, le mouvement féministe, le mouvement autochtone et la contestation des francophones, aussi bien au Québec que hors Québec, de même que les mouvements antiracistes auraient ébranlé le Canada britannique depuis cette décennie charnière au point de créer, selon Palmer, une continuelle situation d'incertitude qui perdure jusqu'à aujourd'hui. Malgré cette description de la complexité des années 1960, Palmer évoque très peu, par des exemples clairs, le lourd héritage de l'Empire britannique et des éléments importants associés à la culture de l'Empire, ce qui l'empêche de voir à quel point certains de ces aspects se sont perpétués et continuent à se perpétuer, comme nous le verrons plus loin, en participant encore grandement aux idéaux de l'Empire britannique dans le processus de construction de la nation canadienne (sur toile de fond britannique). Dans un livre aux prémisses idéologiques et théoriques très

${ }^{4}$ Bryan D. Palmer, Canada's 1960s: The Ironies of Identity in a Rebellious Era, Toronto, University of Toronto Press, 2009. 
différentes, Christian P. Champion ${ }^{5}$ soutient, avec nostalgie, que le Canada a malencontreusement renié ses racines britanniques au cours des années 1960. José Igartua ${ }^{6}$ et Ryan Edwardson ${ }^{7}$ ont, eux aussi, évoqué une " autre Révolution tranquille ", celle du Canada britannique, qui serait passé, dans les années 1960, d'un attachement à l'Empire à un havre multiculturel. Là où Champion déplore l'abandon des traditions britanniques, Igartua et Edwardson laissent clairement entendre que les changements survenus dans les années 1960 ont été superficiels et que certains aspects de la période d'attachement à l'Empire perdurent jusqu'à aujourd'hui, malgré la nouvelle identité canadienne. Nous pensons, comme ces auteurs, que du Canada colonial britannique au Canada postcolonial, la rupture n'a pas été complète et qu'il y aurait, dans certains cas, une étonnante continuité culturelle et politique que, curieusement, la gauche anglophone marxiste ${ }^{8}$, dont Palmer fait partie, et la mouvance postcoloniale anglophone elle-même ont contribué à masquer. Rien n'est plus évident comme exemple de la participation active de la mouvance soi-disant postcoloniale dans le processus de construction nationale conservateur d'aujourd'hui que le livre d'Eve Haque ${ }^{9}$, publié en 2012, sur la commission Laurendeau-Dunton.

Selon Haque, le critère de la langue dans la société canadienne est devenu depuis la Commission royale d'enquête sur le bilinguisme et le biculturalisme un facteur de hiérarchisation raciale entre, d'une part, les Canadiens d'origines française et britannique et, d'autre part,

5 Christian P. Champion, The Strange Demise of British Canada: The Liberals and Canadian Nationalism, 1964-1968, Montréal, McGill-Queen's University Press, 2010.

6 José Igartua, The Other Quiet Revolution: National Identities in English Canada, 1945-1971, Vancouver, University of British Columbia Press, 2006.

7 Ryan Edwardson, Canadian Content: Culture and the Quest for Nationhood, Toronto, University of Toronto Press, 2008.

8 À l'exception de quelques auteurs, dont Ian Angus, Identity and Justice, Toronto, University of Toronto Press, 2008; The Undiscovered Country: Essays in Canadian Intellectual Culture, Edmonton, Athabasca University Press, 2013.

9 Eve Haque, Multiculturalism within a Bilingual Framework: Language Race, and Belonging in Canada, Toronto, University of Toronto Press, 2012. 
les autres, c'est-à-dire les Autochtones et les allophones. Ainsi, la langue des deux groupes fondateurs du Canada serait un facteur culturel important et ne le serait pas pour les locuteurs de langues non officielles. Selon l'auteure, la Commission, ayant reçu un mandat de l'État, a donc mis en œuvre un programme de légitimation des positions respectives des groupes dits fondateurs, contribuant ainsi à la marginalisation de tous les autres. Il en résulterait, selon elle, une égalité des colonialismes dominants à travers une égalité apparente des langues, dorénavant officielles, et une dévalorisation des autres. Que la Commission ait eu beaucoup de difficultés à concilier son mandat en ce qui a trait au biculturalisme avec la réalité multiculturelle déjà fort complexe du Canada ne fait guère de doute. Toutefois, si l'auteure avait voulu être cohérente avec ses propres constats, le livre aurait dû se terminer par un plaidoyer en faveur du multilinguisme et, pourtant, il n'en est rien. Le livre se termine plutôt par la remise en question de l'imaginaire collectif devant mener au désenclavement du bilinguisme et du multiculturalisme. Dans sa perspective, la solution pour sortir le multiculturalisme canadien de l'anémie qui l'afflige réside dans la remise en question du bilinguisme. Le problème avec la thèse de Haque, c'est qu'elle ne tient pas compte du caractère diversifié de la francophonie canadienne, notamment la contribution des immigrants francophones non européens. Dans sa perspective, un francophone canadien est nécessairement un Blanc (une classification, pour dire le moins, problématique si l'on pense, par exemple, à la francophonie canadienne africaine et antillaise, voire à la présence des esclaves noirs et autochtones en Nouvelle-France même ou aux Métis francophones et à Louis Riel).

Moins inquiétante certes, mais néanmoins importante, l'absence de référence dans ce livre au fait que les institutions issues des recommandations de la commission $\mathrm{BB}$, loin de présenter sur un plateau d'argent un accès facile et privilégié aux francophones du Canada à des institutions bilingues, ont laissé les francophones hors Québec sans autre recours qu'un éternel processus de contestation judiciaire afin, justement, d'avoir accès à ces institutions. Comment 
expliquer que Haque se trompe à ce point sur la réalité des francophones du Canada des années 1960 et d'aujourd'hui? La réponse se situe, selon nous, dans la représentation simpliste de la réalité coloniale canadienne que présente cet ouvrage. Si l'auteure rappelle que le colonialisme est une réalité canadienne, cela reste un colonialisme abstrait, sans claire ramification avec la complexité des débats contemporains sur l'Empire britannique. Nous pensons, et c'est ce que nous entendons montrer dans ce qui suit, que cette dimension est, au contraire, fondamentale non seulement pour expliquer le contexte de la commission BB, mais aussi pour comprendre le Canada d'aujourd'hui. Cette intuition est le fil conducteur du texte que le lecteur s'apprête à lire.

Afin de contribuer à mieux comprendre le contexte des années 1960, nous proposons dans les deux prochaines sections un bref aperçu de la littérature récente sur l'Empire britannique et la place que les Canadiens français occupent dans cet Empire. Les Canadiens français auraient été, selon James Kennedy ${ }^{10}$, la première minorité de l'Empire habitant hors des îles britanniques et se sont donc trouvés dans une situation parfois comparable, à plusieurs égards, à celle de la minorité interne de l'Empire au XVIII ${ }^{\mathrm{e}}$ siècle, les Irlandais. Nonobstant le fait que ces deux minorités aient appartenu globalement au monde occidental chrétien et blanc, leur place dans l'Empire a aussi été caractérisée par une continuelle résistance aux tendances à l'uniformisation religieuse et linguistique de l'Empire. Qui plus est, dans le cas des Canadiens français, la Conquête les a coupés de la France métropolitaine et même si leurs élites ont constamment cherché à maintenir des liens avec cette France métropolitaine après la Conquête ${ }^{11}$, ces liens ont été ponctuels, notamment par le

${ }^{10}$ James Kennedy, "Responding to Empire: Liberal Nationalism and Imperial Decline in Scotland and Québec ", Journal of Historical Sociology, vol. 19, $\mathrm{n}^{\circ} 3$ (septembre 2006), p. 284-307, [En ligne], [http://onlinelibrary.wiley.com/ doi/10.1111/j.1467-6443.2006.00283.x/abstract].

11 Gérard Bouchard, Genèse des nations et cultures du Nouveau Monde : essai d'histoire comparée, Montréal, Éditions du Boréal, 2000. Voir aussi Yvan Lamonde et Didier 
recrutement de missionnaires catholiques (on pense, par exemple, aux Oblats). Ainsi, les Canadiens français ont été, d'une certaine façon, coupés de leur métropole coloniale en raison de leur statut de minorité au sein de l'Empire britannique et de la rupture réelle des liens institutionnels continus au XIX ${ }^{\mathrm{e}}$ siècle avec la France. Dans l'évocation du contexte des années 1960, il ne faut jamais négliger cette double coupure, car elle permet de comprendre les raisons pour lesquelles le Canada français a résisté à la culture impériale dominante $^{12}$. Considérer, comme le fait par exemple Eve Haque, les ensembles coloniaux français et britannique comme égaux contribue, selon nous, à masquer le poids encore considérable des réseaux culturels impériaux britanniques, voire à perpétuer leur dominance. Notre thèse est claire : si l'on peut dire du multiculturalisme canadien qu'il est "anémique ", comme le suggère Haque, ce n'est pas parce qu'il est étouffé par le bilinguisme, mais plutôt parce qu'il contribue fortement à masquer les aspects très problématiques de l'héritage colonial britannique et leurs effets, notamment la résistance encore présente au plein déploiement des droits des francophones hors Québec, d'une immigration francophone internationale importante, des droits des Autochtones en matière de revendication territoriale et de la pleine reconnaissance du Québec.

\section{L'Empire et son historiographie}

Selon Tony Ballantyne ${ }^{13}$, l'historiographie portant sur l'Empire britannique a connu au cours des dernières décennies plusieurs transformations, dont la plus importante, à laquelle Alan Lester ${ }^{14}$ et

Poton (dir.), La Capricieuse (1855) : poupe et proue : les relations France-Québec, (1760-1914), Québec, Les Presses de l'Université Laval, 2006.

12 Aspects d'ailleurs évoqués par Yvan Lamonde, Allégeances et dépendances : l'histoire d'une ambivalence identitaire, Montréal, Éditions Nota bene, 2001.

13 Tony Ballantyne, "The Changing Shape of the Modern British Empire and its Historiography ", The Historical Journal, vol. 53, no 2 (juin 2010), p. 429-452, [En ligne], [http://dx.doi.org/10.1017/S0018246X10000117].

${ }^{14}$ Alan Lester, "Imperial Circuits and Networks: Geographies of the British Empire ", History Compass, vol. 4, $\mathrm{n}^{\circ} 1$ (janvier 2006), p. 124-141, [En ligne], [http://onlinelibrary.wiley.com/doi/10.1111/j.1478-0542.2005.00189.x/abstract]. 
Ballantyne lui-même ont contribué de façon significative ${ }^{15}$, a été de le concevoir d'abord et avant tout comme un "réseau culturel». L'histoire de l'effondrement de l'Empire a aussi attiré l'attention des chercheurs qui s'intéressent aux États-Unis (et à l'histoire des Treize Colonies ayant rompu avec l'Empire en 1776), et de ceux dont les travaux portent sur l'Inde (indépendante en 1947) et les colonies africaines (indépendantes dans les années 1960). Même si certains considèrent que, en principe, l'Empire est né avec le Durbar de Delhi le $1^{\text {er }}$ janvier 1877 et le titre d'impératrice des Indes conféré à la reine Victoria, John Darwin dégage, pour sa part, quatre périodes et formes distinctes de l'Empire britannique ${ }^{16}: 1$ ) de 1707 à 1783 ;2) de 1783 à 1815 ; 3) de 1815 à 1857;4) de 1857 aux années 1960 .

Dans le premier Empire britannique (1707 à 1783), la date de 1707 et l'union de l'Angleterre et de l'Écosse indiquent, en fait, que l'Empire britannique fut d'abord un Empire interne ${ }^{17}$, incluant aussi l'Irlande. Cette période aurait été marquée par la construction d'une identité britannique sur le territoire national ${ }^{18}$, selon John Darwin. À l'extérieur, il n'y aurait eu aucune vision impériale unifiée, mais plutôt une multiplicité d'opérations privées menées par différents groupes, principalement des hommes d'affaires et des groupes religieux. Bien que ces groupes aient été protégés par la Royal Navy, et donc par l'État, les historiens du XIX ${ }^{e}$ siècle ont eu tendance à penser que ces Britanniques sont devenus des colonisateurs par accident, en Inde particulièrement où ils auraient constitué un empire malgré eux, absorbés par les affaires politiques de l'Inde alors que leurs seuls intérêts étaient, au départ, commerciaux. L'auteur du XIX ${ }^{\mathrm{e}}$ siècle qui a le plus contribué à diffuser cette thèse fut John Robert

15 Tony Ballantyne, Orientalism and Race: Aryanism in the British Empire, Londres, Palgrave MacMillan, 2002.

16 John Darwin, The Empire Project: The Rise and Fall of the British World-System, 1830-1970, Cambridge, Cambridge University Press, 2009; Unfinished Empire: the Global Expansion, New York, Bloomsbury Press, 2013.

17 John Robertson (dir.), A Union for Empire: Political Thought and the British Union of 1707, Cambridge, Cambridge University Press, 2006.

${ }^{18}$ Linda Colley, Britons: Forging the Nation, 1707-1837, $2^{\mathrm{e}}$ éd., New Haven, Yale University Press, 2009. 
Seeley, auteur de la fameuse phrase «India was waiting to be picked up by somebody ${ }^{19}$ ".

Dans le second Empire britannique, de 1783 à 1815, qui a émergé après la perte des Treize Colonies et jusqu'à la victoire à la fin des guerres napoléoniennes (1815), l'Empire s'est consolidé. Selon Darwin, cette période, bien que courte, a laissé paraître l'une des caractéristiques importantes de l'Empire, à savoir l'adaptabilité de ses agents aux différents contextes. Pendant cette période, la bureaucratie de l'Empire a pris différentes formes, notamment par les relations complexes mais étroites entre l'État et une compagnie comme la Compagnie des Indes orientales. L'expansion impériale, même si elle fut critiquée par Edmund Burke au XvIII ${ }^{\mathrm{e}}$ siècle, William Cobbett au début du XIx et le mouvement radical républicain sous Victoria, a néanmoins produit un vaste sentiment de supériorité culturelle, voire raciale ${ }^{20}$.

La troisième période, de 1815 à 1857 , vit l'acquisition stratégique très importante d'un groupe de petits territoires, notamment les entrepôts commerciaux tels que Hong Kong et Singapour, ainsi que quelques ports isolés en Afrique. Mais l'événement clé de cette période fut l'asservissement économique de la Chine suite aux guerres de l'opium. La période s'est terminée par le désastre de la guerre d'indépendance de l'Inde en 1857, décrite pendant longtemps par l'historiographie britannique comme la Mutinerie indienne. Cette période amena, en apparence, un exemple de cette flexibilité de l'Empire britannique, que mentionne Darwin, caractérisée par une centralisation bureaucratique importante, notamment en Inde, et la dissolution de la Compagnie des Indes orientales.

19 Robert Seeley, Expansion of England, cité dans Nicholas B. Dirks, The Scandal of Empire: India and the Creation of Imperial Britain, Cambridge, The Belknap Press of Harvard University Press, 2006, p. 316.

20 Tony Ballantyne, Webs of Empire: Locating New Zealand's Colonial Past, Wellington, Bridget Williams Books, 2012; Catherine Hall, Civilising Subjects: Metropole and Colony in the English Imagination, 1830-1867, Cambridge, Polity, 2002. 
Ainsi, au cours de la quatrième période, de 1857 aux années 1960, donc la période la plus longue, l'impérialisme libéral de cette époque, fortement teinté de l'influence à long terme de James et John Stuart Mill ${ }^{21}$ et de l'influence de contemporains comme Sir James Fitzjames Stephen, Henry Maine et plusieurs autres, dont Seeley et Cecil Rhodes, fut caractérisé par une expansion en Afrique continentale, des investissements massifs en Amérique du Sud et au Canada, une féodalisation de l'Égypte, en principe possédée par l'Empire ottoman, mais en pratique loué aux Britanniques et aux Français. Le couronnement de Victoria et de ses successeurs lors des Durbars de 1877, 1903 et 1911 furent les faits marquants de l'Empire de cette période avant son déclin graduel au cours de la première moitié du $\mathrm{Xx}^{\mathrm{e}}$ siècle $^{22}$. Mais en 1900, avant le lent déclin des années 1900-1960, l'Empire s'étalait sur un territoire de 12 millions de milles carrés, comprenant un quart de la population mondiale. Entre 1870 et 1900 seulement, l'Empire acquit 4,2 millions de milles carrés de terres et 66 millions de nouveaux sujets de Sa Majesté l'impératrice ${ }^{23}$. C'était plus que tous les autres compétiteurs coloniaux ensemble. Comme Michael Mann a su le montrer ${ }^{24}$, si l'Empire britannique a indéniablement fait partie d'un ensemble colonisateur européen caractérisé à la fois par la compétition et la collaboration occasionnelle, illustrée, par exemple, par la collaboration de la Grande-Bretagne et de la France en Égypte, la place de l'Empire britannique à l'échelle mondiale était nettement hégémonique. Pourtant, malgré cette hégémonie, après la Seconde Guerre mondiale, la Grande-Bretagne, ruinée par la guerre et une mauvaise gestion de son empire, fut incapable de maintenir le système impérial.

${ }^{21}$ Lynn Zastoupil, John Stuart Mill and India, Stanford, Stanford University Press, 1994.

22 Kennedy, "Responding to Empire ».

23 Ibid.

${ }^{24}$ Michael Mann, «Introduction : Empire with Ends », dans Michael Mann (dir.), The Rise and Decline of the Nation-State, Oxford, Blackwell Publishing, 1990; The Sources of Social Power, vol. 2, Cambridge, Cambridge University Press, 1993. 
Or, si la fin de l'Empire fut applaudie par plusieurs en GrandeBretagne même, il ne faut pas oublier que la majorité tenta de résister à son démantèlement. C'est ce que Niall Ferguson ${ }^{25}$ décrit comme le passage de l'arrogance à l'anxiété, mais une anxiété parfois caractérisée par la recrudescence de l'arrogance impériale. Entre 1947 et les années 1960, les Britanniques se retirèrent progressivement de l'Inde et des territoires conquis en Afrique et ailleurs, moins par bienveillance que par nécessité, incapables qu'ils étaient de résister aux pressions des peuples soulevés contre leur hégémonie, surtout en Inde où la pression fut insupportable après 1945. Mais ces retraits furent précédés de politiques répressives, voire de politiques tragiquement cyniques comme la partition de l'Inde. Par ailleurs, on tend aujourd'hui à oublier que le démantèlement de l'Empire donna lieu à des manifestations de violence en Grande-Bretagne contre les travailleurs immigrants asiatiques et africains. En effet, dans les années 1940 et 1950, en Grande-Bretagne, des travailleurs originaires des quatre coins de l'Empire (Caraïbes, Afrique, Pendjab, Bangladesh, Assam, Pakistan) furent l'objet d'attaques en 1948 à Liverpool, en 1949 à Birmingham, Notting Hill et Nottingham en 1958 et Middlesborough en $1961^{26}$. Selon Tony Ballantyne et Wendy Webster ${ }^{27}$, ces attaques contre les immigrants représentaient une résistance de la part des Britanniques au démantèlement de l'Empire, doublée d'une évidente anxiété.

Le Canada de l'époque n'était pas en marge de tous ces changements internationaux des années 1960 et, en particulier, du démantèlement de l'Empire britannique. Le Canada a, lui aussi, ressenti non seulement les révoltes contre la domination impériale qui se déroulaient un peu partout dans le monde - comme l'a si bien évoqué

25 Niall Ferguson, Empire: How Britain made the Modern World, Londres, Penguin, 2003.

${ }^{26}$ Ballantyne, "The Changing Shape of the Modern British Empire and its Historiography".

27 Wendy Webster, Englishness and Empire, 1939-1965, Oxford, Oxford University Press, 2007. 
Herménégilde Chiasson dans son film documentaire sur Louis Robichaud $^{28}$-, mais aussi le contraire de la révolte, c'est-à-dire la résistance au démantèlement de l'Empire. Il ne s'agit pas là d'anecdotes, mais sans doute de la véritable toile de fond de la commission BB. La Commission a été, en quelque sorte, la réponse canadienne à la fin de cet Empire et, avant tout, une réponse aux demandes des Canadiens français, fatigués de l'arrogance de certains Canadiens britanniques, une arrogance qui s'est traduite par une fonction publique fédérale unilingue, une condescendance ostensible vis-à-vis de la soi-disant infériorité économique des Canadiens français, le refus de développer des écoles francophones pour les minorités francophones hors Québec, la domination de l'anglais comme langue de travail au Québec même, etc. Bref, il y a eu dans les années 1960, au Québec et dans les autres communautés, notamment les Acadiens, une opposition au maintien, à l'intérieur du Canada, d'éléments importants de l'Empire, dont le monolinguisme, et, pour reprendre l'expression de l'historien marxiste Stanley B. Ryerson ${ }^{29}$, une révolte face à l'union inégale entre les Canadiens britanniques et les Canadiens français depuis 1867.

Par ailleurs, pour en revenir à l'historiographie portant sur l'Empire britannique, les chercheurs ont accordé, ces dernières années, une attention particulière à ses répercussions sur les peuples autochtones d'Asie et d'Afrique, notamment sur l'économie, la structure sociale, la démographie, la politique et la vision du monde. Un changement de perspective dans l'historiographie a récemment permis d'aborder les questions de langue, de religion, de sexe et d'identité ${ }^{30}$. Les débats récents ont aussi amené les spécialistes à examiner la relation entre la métropole (la Grande-Bretagne elle-même

${ }^{28}$ Herménégilde Chiasson [réalisation, scénario et texte], Robichaud, [enregistrement vidéo], Office national du film du Canada, 1989.58 min $37 \mathrm{s.}$

29 Stanley Bréhaut Ryerson, Unequal Union: Confederation and the Roots of Conflict in the Canadas, 1815-1873, Toronto, Progress Books, 1973.

${ }^{30}$ Darwin, The Empire Project; Unfinished Empire; Ballantyne, "The Changing Shape of the Modern British Empire and its Historiography ». 
et, en particulier, Londres) et les périphéries coloniales. Les historiens du monde britannique insistent sur les aspects matériels, émotionnels et les liens financiers entre les colonisateurs à travers la diaspora impériale, d'où la notion de "réseau culturel impérial ${ }^{31}$ ".

En effet, depuis les années 1990, de nouvelles approches de l'histoire impériale se sont développées et ont donné naissance à ce qui est qualifié de "nouvelle histoire impériale ${ }^{32}$ ". Ces approches ont été caractérisées par deux grandes idées. Tout d'abord, on a avancé que l'Empire britannique était avant tout un projet culturel tout autant qu'un ensemble de relations politiques et économiques. En conséquence, les historiens ont souligné la façon dont la construction de l'Empire a façonné les cultures des peuples colonisés dans l'ensemble britannique même, tout autant que les cultures des dominions blancs, comme le Canada, et des peuples colonisés non blancs. Ils ont montré, en particulier, que l'impérialisme britannique reposait sur les notions de différences culturelles, voire de hiérarchisation des cultures, et que la culture de l'Empire britannique a hiérarchisé races et genres, aussi bien dans les colonies qu'en Grande-Bretagne même. Tout cela ne signifie pas que des phénomènes semblables ne puissent pas être observés chez les autres pays colonisateurs. Mais, comme nous l'avons souligné plus haut, l'Empire britannique a été en position d'hégémonie au $\mathrm{XIX}^{\mathrm{e}}$ siècle et au début $\mathrm{du} \mathrm{Xx}^{\mathrm{e}}$, et le Canada y a joué un rôle important. Pour ne retenir qu'un exemple puisé à même cette nouvelle historiographie de l'Empire britannique, Mrinalini Sinha ${ }^{33}$ a montré comment la prétendue virilité britannique contrastait avec la soidisant mollesse des peuples colonisés ou celle des autres colonisateurs. Des historiens comme Mrinalini Sinha, Antoinette Burton et Catherine Hall ${ }^{34}$ ont utilisé cette approche pour montrer à quel point la culture britannique elle-même a été profondément influencée par

\footnotetext{
31 Lester, "Imperial Circuits and Networks ».

32 Ballantyne, Orientalism and Race.

${ }^{33}$ Mrinalini Sinha, Specters of Mother India: The Global Restructuring of an Empire, Durham, Duke University Press, 2006.

34 Hall, Civilising Subjects.
} 
l'Empire au cours du XIX ${ }^{\mathrm{e}}$ siècle. La deuxième grande tendance qui définit la nouvelle histoire impériale est l'accent mis sur les réseaux institutionnels et culturels qui reliaient les différentes parties de l'Empire. Des travaux plus récents menés par des chercheurs comme Alan Lester et Tony Ballantyne, cités plus haut, ont souligné l'importance de ces réseaux. Ceux-ci ont été constitués de mouvements constants de gens, d'idées, de livres et d'argent qui ont voyagé non seulement entre Londres et les colonies britanniques, mais également directement d'une colonie à l'autre, par exemple, de l'Inde à la Nouvelle-Zélande ou au Canada. Une des prémisses de cette approche est donc que, jusqu'à un certain point, ces réseaux culturels n'auraient pas complètement disparu avec la disparition graduelle de l'Empire et continueraient d'opérer. Selon Robert Young ${ }^{35}$, ce serait encore davantage le cas dans les anciens dominions blancs.

Enfin, pour compléter notre survol de l'historiographie de l'Empire et de sa pertinence pour l'étude du Canada et d'événements comme la commission BB, soulignons la profonde influence de John Greville Agard Pocock dans le renouvellement de l'historiographie britannique. On connaît l'influence qu'a exercée Pocock sur l'historiographie américaine et la science politique avec sa thèse selon laquelle Machiavel, et non John Locke, serait la figure symbolique de la culture politique américaine ${ }^{36}$. Mais au Canada, curieusement, on a tendance à ignorer l'influence de Pocock sur la nouvelle historiographie britannique, sans doute parce que l'on a tendance à ignorer la nouvelle historiographie britannique elle-même ${ }^{37}$. En effet, Pocock, dès les années 1970, a plaidé pour l'importance de renouveler notre compréhension de l'histoire britannique en insistant sur la place de l'Empire ${ }^{38}$.

35 Robert Young, The Idea of English Ethnicity, Malden, Blackwell Publishing, 2008.

36 John Greville Agard Pocock, "British History: A Plea for a New Subject ", The Journal of Modern History, vol. 47, nº 4 (décembre 1975), p. 601-621.

37 Richard Bourke, "Pocock and the Presuppositions of the New British History", The Historical Journal, vol. 53, $\mathrm{n}^{\circ} 3$ (septembre 2010), p. 747-770, [En ligne], [http://dx.doi.org/10.1017/S0018246X10000282].

38 Pocock, "British History ". 
Pour lui, il ne pouvait y avoir de nouvelle historiographie britannique sans une nouvelle histoire de l'Empire. Dans cette perspective, David Armitage $^{39}$ a retracé l'émergence de l'idéologie impériale britannique, de l'époque d'Henri VIII à celle de Robert Walpole dans les années 1720 et 1730. En se basant sur la lecture attentive d'auteurs écossais et irlandais comme Sir Thomas Smith (1513-1577) et David Hume (1711-1776), Armitage fait valoir que l'idéologie impériale devint à la fois un rouage essentiel dans l'unification de l'État britannique et un lien tout aussi essentiel entre celui-ci et les colonies transatlantiques (c'est-à-dire avant la grande expansion en Asie et en Afrique). Armitage relie donc les préoccupations de la "nouvelle histoire britannique " à celles de l'histoire de l'Atlantique et, d'une certaine façon, de la nouvelle historiographie impériale.

Au Canada, nous pensons que ce lien entre la nouvelle histoire britannique et l'histoire de l'Atlantique devrait être fait de façon plus systématique. Par exemple, il n'est pas sans intérêt de remarquer, croyons-nous, qu'une des dimensions fondamentales des années 1960 au Canada a été la contestation de l'Empire, une contestation tous azimuts allant des revendications linguistiques formulées par les francophones du Canada par l'intermédiaire de la commission BB ou, de façon radicale, par le Front de libération du Québec (FLQ), à la contestation des Autochtones, de la Révolution tranquille en Acadie à la contestation socioculturelle de la jeunesse ou, encore, aux émeutes des Noirs lors de la destruction d'Africville à Halifax. Toutes ces manifestations contre les repères identitaires impériaux au Canada ont aussi vivement ébranlé de nombreux Canadiens britanniques, qui ont été de plus en plus nombreux, dans les années 1960, à chercher de nouveaux repères spécifiquement canadiens. Plusieurs responsables politiques à l'époque se sont aussi interrogés sur la nature même du Canada et sur son avenir dans un contexte caractérisé par la croissance économique des États-Unis. La commission Laurendeau-Dunton a

39 David Armitage, The Ideological Origins of the British Empire, Cambridge, Cambridge University Press, 2000. 
été une sorte de catalyseur de ces questionnements sur l'essence du Canada et sa survie. Il est vrai que la Commission n'a pas entièrement répondu à ces interrogations ni exclusivement porté sur les intérêts des francophones, mais elle a été l'expression de l'angoisse qui a accompagné la fin de l'Empire, sans, selon nous, éradiquer complètement l'héritage culturel de la période impériale.

Cet aspect de l'histoire, la contestation de l'Empire britannique, a souvent été voilé par le fait que s'est développée au Canada britannique à partir des années 1950 et au cours des années 1960, sous la plume notamment du philosophe George Grant, une critique de l'autre empire, l'Empire américain, en s'appuyant sur une britannicité supposément anti-impériale ${ }^{40}$. Cette fascination pour l'Empire américain a contribué à masquer la profonde influence de l'Empire britannique au Canada même ${ }^{41}$.

\section{Le Canada et l'Empire}

Comme l'a fait remarquer Colin M. Coates ${ }^{42}$, les Canadiens français ont occupé une place ambiguë dans l'Empire en ce sens qu'ils ont été dans l'Empire sans être de l'Empire. Ils ont ainsi manifesté constamment leur opposition à une conception de l'Empire marquée par une profonde tendance à l'uniformisation culturelle, religieuse et linguistique. Ils ont aussi joué un rôle important, mais pas unique, dans le remplacement des symboles de l'Empire, comme le drapeau et la citoyenneté britannique, sans pour autant complètement réussir à ce que le Canada rompe avec le réseautage culturel et institutionnel

40 Angus, Identity and Justice.

${ }^{41}$ Par ailleurs, il est frappant de remarquer, comme nous le verrons plus loin, que les critiques formulées par Henri Bourassa, dès le début $\mathrm{du} \mathrm{xx}^{\mathrm{e}}$ siècle, dans son ouvrage paru en 1900 et intitulé Great Britain and Canada, à l'égard de l'Empire britannique et de son illégitime mainmise sur le Canada, ont, en fait, trouvé réponse dans les années 1960 avec la commission BB. Voir Henri Bourassa, Great Britain and Canada: topics of the day, Montréal, C. O. Beauchemin, 1901.

42 Colin M. Coates, "French Canadians' Ambivalence to the British Empire ", dans Phillip Buckner (dir.), Canada and the British Empire, Oxford, Oxford University Press, 2008, p. 199. 
de l'Empire. Dès le début du Xx ${ }^{\mathrm{e}}$ siècle, Henri Bourassa ${ }^{43}$ et Olivar Asselin ont tous deux plaidé pour une politique authentiquement canadienne, en rupture avec la politique britannique. Bourassa craignait que le Canada ne se fragmente s'il demeurait au sein de l'Empire britannique, car ses parties iraient inévitablement rejoindre les États-Unis les unes après les autres. Ainsi, au Canada, le nationalisme libéral canadien-français qui s'est développé, selon James Kennedy ${ }^{44}$, au début du $x^{\mathrm{e}}$ siècle, était opposé à l'Empire alors qu'au contraire les Écossais venus au Canada, au demeurant en aussi grand nombre que les Anglais, définissaient leur nationalisme libéral à travers l'Empire, où ils se trouvaient à égalité avec les Anglais, une situation fort différente de celle, beaucoup plus marginale, qui avait cours en Grande-Bretagne même (et cela, malgré l'Acte d'union de 1801, qui avait absorbé notamment l'Irlande). La vision d'Henri Bourassa au sujet du biculturalisme et du bilinguisme, déjà exprimée par George-Étienne Cartier ${ }^{45}$, était difficilement réalisable dans le contexte impérial, caractérisé par la croyance en la supériorité culturelle et religieuse largement partagée par l'élément anglo-saxon. C'était tellement impensable que la résistance à la création de cet espace a continué à être forte dans les années 1950 et les années 1960 pendant les travaux de la commission $\mathrm{BB}$ et continue, pensons-nous, à se manifester, malgré les institutions créées depuis les années 1960,

43 Bourassa, dans d'innombrables écrits, notamment Great Britain and Canada; The Spectre of Annexation and the Real Danger of National Disintegration, Montréal, Le Devoir, 1912; Ireland and Canada, Montréal, Le Devoir, 1914; Le Canada, nation libre? Discours prononcé à Papineauville le 18 juillet 1926, Montréal, Le Devoir, 1926. Voir James Kennedy, Liberal Nationalisms: Empire, State, and Civil Society in Scotland and Québec, Montréal, McGill-Queen's University Press, 2013; "Responding to Empire: Liberal Nationalism and Imperial Decline in Scotland and Québec ", Journal of Historical Sociology, vol. 19, $\mathrm{n}^{\circ} 3$ (septembre 2006), p. 284-307, [En ligne], [http://onlinelibrary.wiley.com/doi/10.1111/ johs.2006.19.issue-3/issuetoc].

44 Kennedy, "Responding to Empire ".

45 Samuel LaSelva, The Moral Foundations of Canadian Federalism: Paradoxes, Achievements, and Tragedies of Nationhood, Montréal, McGill-Queen's University Press, 1996. 
par la résistance, encore aujourd'hui, à la reconnaissance du Québec, à une réelle expansion des communautés francophones hors Québec et des droits des Métis et des Premières Nations. Depuis les années 1960, il y a eu une tendance, dans la littérature, à faire ressortir les aspects du Canada britannique favorables au changement et à l'abandon du passé impérial ${ }^{46}$ au point de faire oublier l'importance de l'attachement à l'Empire et ses effets manifestes encore aujourd'hui.

Certains ouvrages récents sur le Canada mettent en évidence d'autres aspects troublants du lien entre la culture impériale et la xénophobie. Selon James Pitsula ${ }^{47}$, les activités du Ku Klux Klan (KKK) en Saskatchewan et, plus largement, au Canada dans les années 1920 exprimaient justement, de façon radicale, l'attachement de nombreux Canadiens britanniques à l'Empire et, notamment, leur appui à une politique d'uniformisation linguistique. D'abord créé aux États-Unis en 1865 par six soldats de la Confédération sudiste, le KKK fut déclaré hors-la-loi en 1874, mais il refit surface en 1915, notamment à Atlanta et dans le Midwest américain, et, au Canada, à Montréal et en Saskatchewan. Le KKK était non seulement raciste, mais aussi anticatholique, antisémite et violent. Aux États-Unis, le Klan, qui comptait jusqu'à 6 millions de membres entre 1919 et 1925, s'attaqua principalement aux Noirs, aux catholiques, notamment aux catholiques franco-américains du Maine, et aux juifs (le cas le plus spectaculaire étant celui du lynchage de Leo Frank à Atlanta). Les attaques menées par le KKK au Canada auraient, en partie, visé des groupes de non-immigrants, comme les catholiques canadiensfrançais, et des immigrants, notamment ceux originaires d'Europe de l'Est. Bien qu'excessives, ces activités du KKK au Canada

46 Guy Laforest, "Une joute mémorable et ses lendemains : la conférence constitutionnelle de 1968 ", dans Robert Comeau, Michel Lévesque et Yves Bélanger (dir.), Daniel Johnson : rêve d'égalité et projet d'indépendance, Québec, Presses de l'Université du Québec, 1991.

${ }^{47}$ James M. Pitsula, Keeping Canada British: The Ku Klux Klan in 1920s Saskatchewan, Vancouver, University of British Columbia Press, 2013. 
exprimaient, selon Pitsula, l'opinion d'une vaste majorité de Canadiens britanniques et leur attachement à une société protestante, uniquement de langue anglaise et fleurissant dans un empire dont personne ne prévoyait la fin dans les années 1920 et 1930, pas même ceux qui, à l'intérieur de l'Empire, s'y opposaient. Comme nous l'avons déjà fait remarquer, la majorité des Britanniques et peut-être, en particulier, les Britanniques des dominions, n'ont pas pour autant compris que la fin de la Seconde Guerre mondiale annonçait la disparition inévitable de l'Empire. De plus, au Canada, comme l'ont montré José Igartua $^{48}$, George Richardson et Paul Rutherford ${ }^{49}$, l'emprise culturelle et politique de la Grande-Bretagne impériale a continué au-delà des années 1950 et 1960, en particulier dans les programmes destinés aux écoles primaires et secondaires, dans lesquels la grandeur de l'Empire et son rôle civilisateur ont continué à être décrits sans critique et avec beaucoup d'enthousiasme.

Faut-il alors s'étonner si, dans le contexte des années $1960 \mathrm{au}$ Canada, il y a eu une réaction hostile face à la demande du Canada français d'établir un partenariat d'égal à égal entre francophones et anglophones? Il serait sans doute naïf d'affirmer que les principes du bilinguisme ont été accueillis unanimement par la majorité canadienne britannique. Comme l'ont rappelé Marcel Martel et Martin Pâquet ${ }^{50}$, l'ancien député, ministre fédéral et juge, Joseph T. Thorson a sillonné le pays après 1969 pour empêcher l'adoption de la Loi sur les langues officielles et s'opposer au bilinguisme au Nouveau-Brunswick. Sa cause a finalement été entendue en 1974 par la Cour suprême du Canada, qui a rejeté sa demande d'injonction contre la proclamation de deux langues officielles au Canada et au Nouveau-Brunswick. Mais trois

48 Igartua, The Other Quiet Revolution.

49 George Richardson, "Nostalgia and National Identity: The History and Social Studies Curriculum of Alberta and Ontario at the End of Empire ", dans Phillip Buckner (dir.), Canada and the End of Empire, Vancouver, University of British Columbia Press, 2004, p. 249-267; et Paul Rutherford, "The Persistence of Britain: The Culture Project in Post-War Canada ", dans Ibid., p. 195-205.

50 Marcel Martel et Martin Pâquet, Langue et politique au Canada et au Québec: une synthèse historique, Montréal, Éditions du Boréal, 2010. 
ans plus tard, en 1977, l'historien conservateur Donald Creighton a repris la lutte en publiant des articles incendiaires contre le concept de biculturalisme, niant avec véhémence la pertinence de la thèse des deux peuples fondateurs et s'attaquant, dans le magazine Maclean's, à la Charte de la langue française du Parti québécois ${ }^{51}$. Ces quelques exemples montrent bien la résistance importante au bilinguisme. Ainsi, jusqu'à aujourd'hui, les réformes issues des travaux de la commission BB, loin de créer l'unanimité chez les peuples fondateurs, n'ont cessé d'être remises en question, notamment par les partisans sans doute nostalgiques de l'Empire, où l'hégémonie de la langue anglaise était incontestable. En Grande-Bretagne même, comme nous l'avons présenté ci-dessus, l'idée que plusieurs institutions, certains réseaux nationaux et internationaux créés à l'époque de l'Empire et certains traits culturels de cette époque persistent encore aujourd'hui est largement acceptée. Nous pensons que cette idée doit être étendue à l'étude du Canada.

\section{Un bilan?}

En somme, malgré tous les changements qui ont eu lieu au cours des dernières décennies et la redéfinition apparente du Canada, plusieurs aspects du Canada d'aujourd'hui ont un air de parenté avec la période qui a marqué la fin de l'Empire durant les années 1950 et 1960. Malgré les nombreuses mesures mises en place depuis quarante ou cinquante ans, la situation des francophones hors Québec fait toujours l'objet, au mieux, d'une indifférence collective, voire, dans certains cas, d'une résistance hostile, notamment en Alberta. Par ailleurs, le dossier des revendications territoriales des Autochtones n’a guère bougé de même que celui des Métis. Enfin, la reconnaissance politique et constitutionnelle du Québec est toujours dans l'impasse. Comme on peut le voir dans la chronologie présentée ci-dessous, les changements institutionnels qui ont été apportés à la suite de la

51 Ibid. 
commission Laurendeau-Dunton n'ont pas procuré aux francophones hors Québec un accès plus important aux institutions. Au contraire, cet accès a souvent été rendu possible uniquement à la suite de contestations judiciaires, une réalité qui perdure depuis plus de cinquante ans. De plus, le bilan démographique de ces décennies, voire depuis 1931, n'est pas tellement positif pour les communautés francophones hors Québec.

La liste suivante illustre d'ailleurs, à l'aide de quelques événements importants concernant les droits des francophones hors Québec, le dédale juridique dans lequel les francophones se sont trouvés pour avoir accès à des institutions et des services pourtant reconnus par différentes lois ou par la Loi constitutionnelle de 1982.

\section{Tableau 1}

\section{Événements importants concernant les droits des francophones hors Québec}

\begin{tabular}{|l|l|}
\hline 1988 & L'arrêt Mercure pour l'Alberta et la Saskatchewan. \\
\hline 1990 & $\begin{array}{l}\text { Le jugement de la Cour suprême du Canada dans l'affaire } \\
\text { Mahé. }\end{array}$ \\
\hline $\mathbf{1 9 9 6}$ & $\begin{array}{l}\text { Une innovation en gouvernance paritaire apparaît avec } \\
\text { la création du Comité national de développement des } \\
\text { ressources humaines de la francophonie canadienne. }\end{array}$ \\
\hline 2099 & $\begin{array}{l}\text { L'arrêt Beaulac de la Cour suprême du Canada précise que } \\
\text { l'égalité des langues officielles du Canada signifie l'accès } \\
\text { égal à des services de qualité égale. }\end{array}$ \\
\hline 2001 & $\begin{array}{l}\text { L'arrêt Arsenault-Cameron de la Cour suprême du Canada } \\
\text { précise les pouvoirs des conseils scolaires et les obligations } \\
\text { des gouvernements. }\end{array}$ \\
\hline $\begin{array}{l}\text { Un ministre responsable des langues officielles est nommé } \\
\text { pour la première fois. }\end{array}$ \\
\hline $\begin{array}{l}\text { Dans la Loi sur l'immigration et la protection des réfugiés, les } \\
\text { langues officielles font partie des critères dont il faut tenir } \\
\text { compte dans le recrutement des immigrants. }\end{array}$ \\
\hline
\end{tabular}




\begin{tabular}{|l|l|}
\hline 2003 & $\begin{array}{l}\text { Dans le jugement Doucet-Boudreau c. Nouvelle-Écosse, } \\
\text { la Cour suprême du Canada confirme que les tribunaux } \\
\text { doivent accorder des réparations en fonction de l'objet du } \\
\text { droit et qu'ils peuvent assurer un suivi pour veiller à ce } \\
\text { que la réparation soit complète et efficace. }\end{array}$ \\
& $\begin{array}{l}\text { Le gouvernement de Jean Chrétien annonce son Plan } \\
\text { d'action pour les langues officielles. }\end{array}$ \\
\hline 2003-2014 & $\begin{array}{l}\text { L'affaire Caron en Alberta. Le jugement favorable accordé } \\
\text { à Gilles Caron par la Cour provinciale a été invalidé par la } \\
\text { Cour du Banc de la Reine de l'Alberta et la Cour d'appel } \\
\text { de l'Alberta. Cette cause a été entendue en Cour suprême. }\end{array}$ \\
\hline
\end{tabular}

Parallèlement au dédale juridique complexe qui a maintenant épuisé au moins deux générations de francophones hors Québec, les quelques chiffres suivants montrent, selon nous, à quel point, sur les plans démographique et linguistique, rien n'a fondamentalement changé au Canada depuis les années $1960^{52}$.

- $\quad$ En 1931, en ce qui a trait aux deux langues officielles du Canada, $68 \%$ des Canadiens ne parlaient que l'anglais et $18 \%$ ne parlaient que le français. En 2011, soit 80 ans plus tard, rien n'a vraiment changé puisque $68 \%$ des Canadiens ne parlent toujours que l'anglais, et $12 \%$ ne parlent que le français; donc la situation de l'anglais n'a pas bougé alors que celle du français a connu un recul. Le graphique, en annexe, illustre bien cette constante : la ligne droite tracée de 1931 à 2011, indique clairement que la proportion des Canadiens qui ne parlent que l'anglais n'a pas bougé au cours de cette période.

52 Statistique Canada, 2011 Census of Population : Linguistic Characteristics of Canadians, Catalogue n ${ }^{\circ}$ 98-314-X2011001, [En ligne], [http://www12.statcan. gc.ca/census-recensement/2011/as-sa/98-314-x/98-314-x2011001-eng.pdf] (24 mars 2013); Languages Reference Guide 2011, Catalogue n 99-010-XWE2011007, [En ligne], [http://www12.statcan.gc.ca/nhs-enm/2011/ref/guides/99-010-x/99010-x2011007-eng.cfm] (27 juin 2013). 
- En 2011, 17,5\% des Canadiens parlent les deux langues officielles (17,4\% en 2006). Mais cette dernière statistique représente essentiellement le taux de bilinguisme au Québec. Dans cette province, en 1951, 26 \% de la population était bilingue, alors que $42,5 \%$ parlait les deux langues en 2011. Aujourd'hui, 66,1\% des anglophones du Québec sont bilingues, alors que $37 \%$ des francophones et 50,4\% des allophones sont bilingues. Dans le reste du Canada, 7,1 \% des anglophones sont bilingues, $85,1 \%$ des francophones et seulement 5,7 \% des allophones le sont. Au total, donc, $44 \%$ des francophones du Canada sont bilingues et seulement $9 \%$ des anglophones le sont.

Ces chiffres dépeignent, selon nous, un Canada qui, malgré l'apparition d'indices favorables au bilinguisme et, de façon embryonnaire, au multilinguisme, est encore largement caractérisé par la domination et l'hégémonie de l'anglais, sauf évidemment au Québec où la force d'attraction de l'anglais est néanmoins toujours présente. Au Québec, la région de Montréal semble être la véritable capitale du bilinguisme, voire du multilinguisme où, comme le montrent les statistiques de 2011, le taux de rétention des langues autres que le français et l'anglais est plus élevé qu'ailleurs au Canada. En effet, c'est au Québec, selon le recensement de 2011, qu'a eu lieu la plus forte augmentation de gens déclarant parler à la maison une langue non officielle. En fait, lorsque la langue officielle parlée à la maison au Québec est le français, le pourcentage de gens déclarant parler aussi à la maison une autre langue non officielle est plus élevé. Ainsi, au Québec selon le recensement de $2011^{53}$, le pourcentage de Québécois déclarant parler uniquement le français à la maison est passé, depuis 2006, de 75,1 \% à 72,8\%. Mais la proportion de Québécois déclarant parler à la maison le français et une autre langue non officielle est

53 Ibid. 
passé, entre 2006 et 2011, de 3,8 \% à $5 \%$, alors que la proportion de Québécois déclarant parler l'anglais et une autre langue non officielle est passé de 2,5\% à 2,8 \% ${ }^{54}$. Il semble donc qu'au Québec, le multilinguisme soit lié, notamment à Montréal, à un contexte où plus de gens parlent les deux langues officielles. C'est donc au Québec et, surtout, à Montréal que ce serait réalisé l'idéal canadien défini par les événements des années 1960 et l'influence de la commission Laurendeau-Dunton, alors que le reste du Canada ressemble étrangement, malgré certaines apparences, au Canada de la fin de la période impériale.

\section{Conclusion}

Si l'on devait relancer une autre commission d'enquête sur le bilinguisme au Canada, ce serait important de rappeler que, dans le contexte canadien, une langue comme le français peut être une source de résistance contre le colonialisme, même si, évidemment, le français a lui-même été une langue coloniale. Selon nous, cinquante ans après la commission BB, le Canada britannique sur fond de passé colonial se perpétue lentement mais sûrement. Il est fascinant de constater que depuis les années 1960 et 1970, même après de nombreuses victoires juridiques auprès de la Cour suprême, comme nous l'avons déjà fait remarquer, la situation des revendications territoriales des Autochtones et celle des francophones hors Québec n'a pas fondamentalement changé.

Malgré leur critique acerbe du legs du passé colonial dans le Canada contemporain, des auteurs comme Eve Haque n'ont pas su analyser la hiérarchie dans les relations de pouvoir au sein même des anciennes puissances coloniales. Situer dans son contexte historique global l'analyse de cette hiérarchie devrait permettre de mieux voir la permanence de certains traits de l'Empire britannique et sa reproduction culturelle, tout en donnant une voix à des acteurs

${ }^{54}$ Ibid. 
largement oubliés dans les grands débats des dernières années, comme la francophonie non blanche, qu'elle soit autochtone, métisse, antillaise et africaine. Rappelons aussi que depuis les années 1970, seulement $3 \%$ des immigrants au Canada sont francophones, un déséquilibre qui perdure au détriment, notamment, des francophonies antillaise et africaine, et qui mériterait qu'on s'y attarde, car il s'agit peut-être d'un autre aspect voilé de l'héritage colonial britannique et de son projet d'exercer son hégémonie culturelle sur des francophones. Dans Genuine Multiculturalism: The Tragedy and Comedy of Diversity, Cecil Foster écrit : "Tragically, the only hope for a new Canada would come not from the preservation of either of the traditional ethnic nations in Canada - but from the death and rebirth of both as a single federal state ${ }^{55}$."Selon nous, ce seul espoir réside, en fait, dans la déconstruction du passé colonial et impérial, trop facilement oublié.

55 Cecil Foster, Genuine Multiculturalism: The Tragedy and Comedy of Diversity, Montréal, McGill-Queen's University Press, 2014, p. 25. 


\section{Annexe}

\section{Graphique 1}

Pourcentage de la population canadienne pouvant parler l'anglais, le français, les deux langues officielles ou seulement une ou plusieurs langues non officielles, 1931-2011

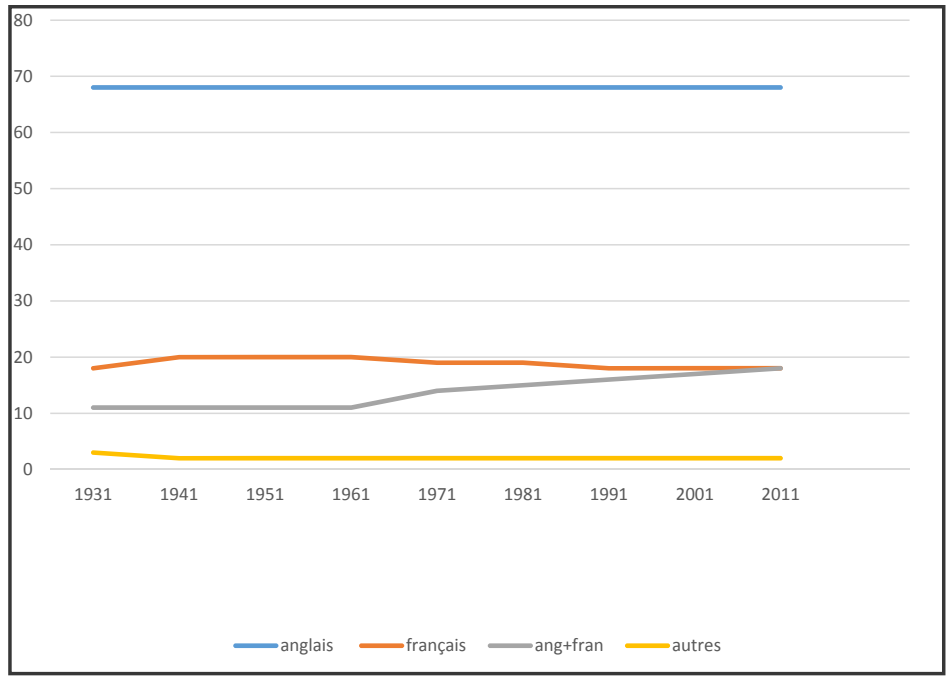

Sources : Graphique établi à partir des données recueillies par Statistique Canada, 2011 Census of Population : Linguistic Characteristics of Canadians, Catalogue n ${ }^{\circ}$ 98-314-X2011001, [En ligne], [http://www12.statcan.gc.ca/censusrecensement/2011/as-sa/98-314-x/98-314-x2011001-eng.pdf]; Languages Reference Guide 2011, Catalogue n ${ }^{\circ}$ 99-010-XWE2011007, [En ligne], [http:// www12.statcan.gc.ca/nhs-enm/2011/ref/guides/99-010-x/99-010-x2011007eng.cfm]. 